\title{
Wing and Body Kinematics of Takeoff and Landing Flight in the Pigeon (Columba livia)
}

\section{Citation}

Berg, Angela M. and Andrew A. Biewener. 2010. Wing and body kinematics of takeoff and landing flight in the pigeon (Columba livia). Journal of Experimental Biology 213(10): 1651-1658.

\section{Published Version}

doi:10.1242/jeb.038109

\section{Permanent link}

http://nrs.harvard.edu/urn-3:HUL.InstRepos:4894724

\section{Terms of Use}

This article was downloaded from Harvard University's DASH repository, and is made available under the terms and conditions applicable to Open Access Policy Articles, as set forth at http:// nrs.harvard.edu/urn-3:HUL.InstRepos:dash.current.terms-of-use\#OAP

\section{Share Your Story}

The Harvard community has made this article openly available.

Please share how this access benefits you. Submit a story.

\section{Accessibility}




\section{Wing and body kinematics of takeoff and landing flight in the pigeon (Columba livia)}

Takeoff and landing are critical phases in a flight. To better understand the functional importance of the kinematic adjustments birds use to execute these flight modes, we studied the wing and body movements of pigeons (Columba livia) during short-distance free-flights between two perches.

8 The greatest accelerations were observed during the second wingbeat of takeoff. The wings were responsible for the majority of acceleration during takeoff and landing, with the legs contributing only one-quarter of the acceleration. Downstroke amplitude, wingbeat frequency, and downstroke velocity were all greatest during takeoff flight and decreased with each successive takeoff wingbeat. These three parameters, which relate to aerodynamic power output, increased during landing flight until the final wingbeat, when they decreased. These patterns indicate that downstroke velocity must be greater

14 for slower flight to increase the amount of air accelerated by the wings, regardless of whether the bird is increasing or decreasing its flight velocity. Pigeons used multiple mechanisms to adjust thrust and drag to accelerate during takeoff and decelerate during landing. Body angle, tail angle, and wing plane angles all shifted from more horizontal orientations during takeoff to near-vertical orientations during landing, thereby reducing drag during takeoff and increasing drag during landing. The stroke plane was tilted steeply downward throughout takeoff (increasing from $-60 \pm 5^{\circ}$ to $-47 \pm 1^{\circ}$ ), supporting our hypothesis that a downward-tilted stroke plane pushes more air rearward to accelerate the bird forward. Similarly, the stroke plane tilted upward during landing (increasing from $-1 \pm 2^{\circ}$ to $17 \pm 7^{\circ}$ ), implying that an upward-

22 tilted stroke plane pushes more air forward to slow the bird down. Rotations of the stroke plane, wing planes, and tail were all strongly correlated with rotation of the body angle, suggesting that pigeons are 
able to redirect aerodynamic force and shift between flight modes through modulation of body angle 2 alone.

\section{INTRODUCTION}

For most bird species, flying is an essential part of each individual's daily life. Birds fly to forage, to evade predators, to migrate, and for numerous other reasons. To be successful, birds not only need to be able to stay aloft, they must be able to takeoff from a stationary position and land without injury

8 at the end of the flight. The ease with which most birds accomplish these tasks has, however, resulted in comparatively little study relative to the considerable attention that has been devoted to understanding fundamental aspects of steady forward flight. A successful takeoff involves leaping into the air and accelerating to the desired flight speed. When landing, a bird must not slow down too much

12 too early, or it will fall from the air prematurely. Yet, a bird must decelerate enough to be able to gauge the mechanical properties of a perch and to absorb the body's remaining kinetic energy with its legs

14 when it encounters the perch.

Kinematics analysis of takeoff and landing in previous work has largely been limited to wingbeat frequency and amplitude (Dial, 1992), general descriptions of the wingstroke (Brown, 1948; Simpson, 1983), or has focused on only one flight mode, hindering comparison among flight modes (Green and Cheng, 1998; Earls, 2000; Tobalske and Dial, 2000; Tobalske et al., 2004). In the rock pigeon (Columba livia, Gmelin 1798), wingbeat frequency has been found to be similar during level flight and landing, but greater during takeoff (Dial, 1992). In the same study, wingbeat amplitude was higher during takeoff and landing, compared to level flight. The present study explores more broadly and in more detail the

22 kinematic parameters of both takeoff and landing, with the goal of identifying the wing and body kinematics that produce accelerating and decelerating flight. 
Most previous work on takeoff and landing has focused on muscle function and power

2 requirements (e.g. Dial, 1992; Biewener et al., 1998; Tobalske and Dial, 2000; Askew et al., 2001;

Usherwood et al., 2005). Several of these studies have compared takeoff and landing in order to explore

4 the range of flight muscle function. In pigeons, several wing muscles show the strongest activation during takeoff from the ground or landing on a perch (Dial, 1992). However, compared to midflight, the large, anterior sternobrachial region of the pectoralis (the main downstroke muscle) performs less work during takeoff from a perch and more work during the initial phases of landing flight (Biewener et al., 1998). The supracoracoideus (the main upstroke muscle) also generates lower stress and fascicle strain, and thus less power, during takeoff and landing (Tobalske and Biewener, 2008). Similarly, consistent with theory, differential pressure measurements suggest greater aerodynamic power production at distal regions of the wing during takeoff and landing, when birds are flying slowly and accelerating or 12 decelerating (Usherwood et al., 2005).

Intrinsically, takeoff and landing involve changes in flight speed. Previous studies have more often examined the kinematics and power requirements when birds fly steadily over a range of speeds in a wind tunnel (Tobalske and Dial, 1996; Tobalske et al., 2003; Hedrick et al., 2004; Rosen et al., 2004). These studies have shown that in several bird species, wingbeat frequency varies little across flight speeds. However, body angle decreases with increasing speed in several species (Tobalske and Dial, 1996; Tobalske et al., 2003; Hedrick et al., 2004). Tobalske, et al. found that stroke plane angle becomes more vertical and wing excursion increases with increasing flight speeds in budgerigars (Melopsittacus undulates), cockatiels (Nymphicus hollandicus), ring-neck doves (Streptopelia risoria), and black-billed magpies (Pica hudsonia), (Tobalske et al., 2003). In contrast, Rosen, et al. found that the inclination

22 angle of the wingtip path in a thrush nightingale (Luscinia luscinia) becomes steeper with decreasing flight speeds, and that wingbeat amplitude does not depend on flight speed (Rosen et al., 2004). These 24 differing results indicate that species can differ in these kinematic flight parameters. Familiarity with a 
perch can also affect the speed at which a bird will land. Green and Cheng found that pigeons approach

2 more quickly as they become familiar with a perch (Green and Cheng, 1998).

This paper presents the first of a series of investigations we have undertaken to analyze the

4 mechanisms of takeoff and landing flight in birds. The aim of this study was to understand the underlying kinematics of takeoff and landing maneuvers in a generalist flyer. Pigeons were conditioned to fly between two perches while their movements were recorded with high-speed video. Because takeoff and landing require accelerations in opposite directions, we expected to observe vastly different

8 kinematics between these two flight modes. To accelerate forward during takeoff, we expected birds to exhibit greater wing stroke amplitudes in order to accelerate more air. We also expected the tail to be narrower during takeoff in order to reduce drag. We expected the stroke plane angle to be tilted steeply downward during takeoff to accelerate more air rearward and the bird forward. In contrast, 12 during landing flight, we expected the stroke plane angle to be tilted upward to accelerate more air forward, thereby slowing the bird down. Compared to takeoff and midflight, we expected faster wing velocity during landing flight in order to increase drag on the wing and assist in decelerating the bird.

\section{METHODS}

\section{Animals and flight arena}

Rock pigeons were housed at the Concord Field Station in Bedford, MA, USA, and provided with food and water ad libitum. Birds were trained regularly to fly between two perches 6-8 $\mathrm{m}$ apart. The confounding effects of a pigeon encountering a novel perch (Green and Cheng, 1998). The perches were

22 made of wood dowels $2.5 \mathrm{~cm}$ in diameter, covered with tape to provide a gripping surface for the birds, and mounted on wooden stands $1 \mathrm{~m}$ tall. 


\section{Kinematic data collection}

Data from seven birds ( $431 \pm 67 \mathrm{~g}$, mean body mass \pm standard deviation) were used to obtain detailed kinematics. Three to four high-speed digital cameras were positioned around the flight corridor. Depending on circumstances, one Photron FastCam-X $1280 \mathrm{PCl}$ camera, one or two Photron FastCam 1024 PCl cameras (Photron USA Inc., San Diego, CA, USA), and zero to two RedLake PCI 500 cameras (RedLake Inc., San Diego, CA, USA) were used. Cameras recorded at 250 frames s$^{-1}$ with shutter speeds of $1 / 500$ to $1 / 2000$ s. Cameras were calibrated using the direct linear transform method (Hatze, 1988) and the MatLab ${ }^{\mathrm{TM}}$ (version 7.4; 2007, The MathWorks, Inc., Natick, MA, USA) script DLTcalibration.m written by Ty Hedrick (Hedrick, 2008). All axes were defined in the global reference frame. The $x$-axis was defined as the axis along the flight corridor; the $y$-axis as perpendicular to the $x$-axis and in the horizontal plane; and the $z$-axis as the vertical. anatomical landmarks: wing root (as proxy for the shoulder), wrist, wingtip ( $9^{\text {th }}$ primary feather), rump, and three points on the trailing edge of the tail (Fig. 1A). Birds 1-5 were also marked on the $6^{\text {th }}$ primary feather and $2^{\text {nd }}$ secondary feather. The straightest, most level flights for each bird were selected for analysis. After data collection, the marked points on the wing nearest the camera were digitized using the MatLab ${ }^{\mathrm{TM}}$ script DLTdv3.m written by Ty Hedrick (Hedrick, 2008). Position data was filtered at four times the average wingbeat frequency for the filmed portion of the flight. Three takeoff flights and three landing flights were analyzed for Birds 1-5. For this study, four mid-flights were analyzed for Birds $2,4,6$, and 7.

\section{Kinematic calculations}

Wingbeat phase was determined by the position of the wingtip in the $y$-axis, relative to the wing root. The upstroke-downstroke transition (USDS) and the downstroke-upstroke transition (DSUS) were 
designated as the times when the wingtip was most medial to the wing root, and dorsal or ventral to the

2 bird's body, respectively. One wingbeat was designated as the time from one USDS to the next USDS. Mid-downstroke was designated as the time when the wingtip was farthest from the wing root in the $4 \quad y$-direction during the downstroke.

Because the birds did not always touch down onto the perch at the same wingbeat phase, it was necessary to standardize the wingbeat numbers relative to the moment the feet touched the perch ("footdown"). The zeroth wingbeat was defined as the wingbeat that ended either after footdown, or 8 less than 20 ms before footdown. In other words, the USDS that occurred after footdown or less than 20 ms before footdown defined the end of "wingbeat 0. " The wingbeats were then counted backwards in time as wingbeats $-1,-2$, and -3 . During takeoff, birds consistently left the perch after the first USDS and before the first mid-downstroke. This first USDS defined the beginning of wingbeat 1 , which was 12 followed by wingbeats 2,3 , and 4.

The mid-back of the bird was approximated by using the $y$-coordinate of the rump and the average of the $x$-coordinates of the rump and wing root. This point served as a proxy for the center of mass, which was not measured on these birds. Whole-body horizontal velocity $\left(\mathrm{V}_{x}\right)$ was calculated by differentiating mid-back position data along the $x$-axis. $V_{x}$ values were averaged from one time step before USDS to one time step after USDS to obtain a mean $\mathrm{V}_{x}$ at USDS. The difference between the mean $V_{x}$ values at sequential USDSs gave the whole-body average horizontal acceleration over the wingbeat $\left(A_{x, w b}\right)$.

Stroke plane angles (SPAs) were calculated using the wingtip path projected onto the $x-z$ plane. Because the SPAs changed sign during the flights, SPAs were calculated as the arctangent of the slope of 22 the linear regression of the projected path, with the sign as indicated in Fig. 1B (a downward-tilted stroke plane corresponding to a negative SPA, and an upward-tilted stroke plane corresponding to a positive SPA). The local SPA (SPA $A_{\text {loc }}$ ) used the wingtip path relative to the wing root for the entire 
wingbeat. The global downstroke plane angle $\left(\mathrm{DSPA}_{\text {glob }}\right)$ used the wingtip path in the global coordinate

2 system of the downstroke only. Body angle was calculated as the angle between the horizontal and the line connecting the wing root and the rump, also projected onto the $x-z$ plane. Protraction was

4 calculated as the distance from the $\mathrm{SPA}_{\text {loc }}$ to the wing root in the $x-z$ plane.

The wing markers on Birds 1-5 allowed three distinct wing planes to be analyzed: the arm wing, the proximal hand wing, and the distal hand wing, as illustrated in Fig. 1A. The vertical angle of these wing planes was determined as the angle between the plane and the horizontal. Tail angle was also

8 calculated as the angle between the tail plane and the horizontal. The tail plane was defined by the three marks on the trailing edge of the tail. Because of the mobility of the tail relative to the rump mark, the rump was not used to determine tail spread. Instead, the trailing edge marks were used to determine the tail spread using the following method. Because the trailing edge of the tail feathers

12 form a nearly circular arc when spread, the tail was assumed to be a portion of a circle (Fig. 1A). The radius, $R$, of this circle was determined using the equation:

$$
R=\frac{a \cdot b \cdot c}{\sqrt{2 a^{2} b^{2}+2 b^{2} c^{2}+2 c^{2} a^{2}-a^{4}-b^{4}-c^{4}}}
$$

14 where $a, b$, and $c$ are each a distance between two of the trailing edge marks (Fig. 1A). The tail spread was then calculated as:

$$
\text { spread }=2 \sin ^{-1} \frac{a}{2 R}+2 \sin ^{-1} \frac{b}{2 R}
$$
using two terms with $a$ and $b$ (instead of one term with $c$ ) in order to permit values greater than $180^{\circ}$.

Downstroke sweep angle $(\theta)$ is a measure of stroke amplitude that accounts for angular motion of the stroke. The angle through which the wingtip swept at each time step was summed over the entire downstroke to give $\theta$. The value of $\theta$ was divided by the duration of the downstroke to give the downstroke sweep velocity $(\omega)$.

Kinematic analyses were performed using custom MatLab ${ }^{\mathrm{TM}}$ scripts. 


\section{Statistical analysis}

For each flight, the wingbeats were numbered as described above. For each bird, parameter

4 values for wingbeats of the same number were averaged among the flights for that bird. This procedure gave series of mean values to describe the numbered wingbeats of each bird. Mean values for wingbeats of the same number were averaged among all birds, giving the grand mean for each parameter for each wingbeat number. Unless otherwise noted, values reported are grand means.

8 Repeated-measures analyses by both bird and wingbeat were performed in SPSS (version 16.0; SPSS Inc., Chicago, IL, USA). Linear regressions and $t$-tests were performed in Microsoft Excel (2007; Microsoft Corp., Redmond, WA, USA). To account for multiple comparisons, the sequential Bonferroni method (Rice, 1989) was used to test for significance of differences observed in sets of repeated-measures analyses and in sets of post hoc $t$-tests. Values are reported as mean \pm standard deviation.

\section{RESULTS}

Along the portions of the flight path analyzed for takeoff, most birds continuously increased their horizontal velocity (Fig. 2A, supplementary material Movie 1). Likewise, in the portion of landing analyzed, most birds continuously decreased their velocity (supplementary material Movie 2). Whole-body velocity varied within individuals more during landing than during takeoff. During takeoff, the horizontal velocity $\left(V_{x}\right)$ was $1.52 \pm 0.23 \mathrm{~m} \mathrm{~s}^{-1}$ at the beginning of wingbeat 1 , when the feet were

22 leaving the perch; and $4.51 \pm 0.48 \mathrm{~m} \mathrm{~s}^{-1}$ at the end of wingbeat 4 (Fig. 2B). During landing, $\mathrm{V}_{\mathrm{x}}$ was $4.19 \pm 0.27 \mathrm{~m} \mathrm{~s}^{-1}$ at the beginning of wingbeat -3 , and $0.97 \pm 0.32 \mathrm{~m} \mathrm{~s}^{-1}$ at the end of wingbeat 0 . 
Acceleration in the $x$-direction over the course of the wingbeat $\left(A_{x, w b}\right)$ was greatest for wingbeat

$22\left(9.14 \pm 2.22 \mathrm{~m} \mathrm{~s}^{2}\right)$ during takeoff and decreased during subsequent wingbeats (Fig. 2B). During landing, $A_{x, w b}$ became more negative, with wingbeat 0 exhibiting the most negative $A_{x, w b}\left(-8.50 \pm 2.52 \mathrm{~m} \mathrm{~s}^{2}\right)$. As

4 expected, kinetic energy (KE) followed $V_{x}$, increasing during takeoff and decreasing during landing (Fig. 2C). Because the most level flights between the perches were selected for analysis, potential energy

6 (PE) did not change significantly during the takeoffs or landings (repeated-measures, $F=1.778, P=0.117$;

Fig. 2C). Generally, the pigeons did not land from distances sufficiently above or below the perch to 8 alter their PE under the experimental flight conditions.

Body angle fluctuated during each wingbeat, reaching its steepest angle at or just after the upstroke-downstroke transition (USDS; supplementary material Movies 1-3). During takeoff, the body angle was shallowest near the end of downstroke. During landing and midflight, the body angle was

12 shallowest near mid-downstroke. Body angle at mid-downstroke $\left(\mathrm{BA}_{\mathrm{mds}}\right)$ increased dramatically over the course of the flight (Fig. 3). $\mathrm{BA}_{\mathrm{mds}}$ averaged $-13.0 \pm 7.9^{\circ}$ for wingbeat 1 , indicating that the wing root was below the rump for the first wingbeat. During midflight, $B A_{m d s}$ was $19.0 \pm 9.3^{\circ}$, and by wingbeat $0, B A_{m d s}$ was $72.0 \pm 7.8^{\circ}$. Birds occasionally pitched beyond the vertical during the final landing wingbeat, resulting in a body angle that exceeded $90^{\circ}$.

Tail angle also fluctuated during each wingbeat. Although the timing patterns of maximum and minimum tail angle varied among individuals, for landing it was frequently observed that the tail was at its steepest at the downstroke-upstroke transition (DSUS) and at its shallowest during mid-downstroke. Like body angle, tail angle at mid-downstroke $\left(\mathrm{TA}_{\mathrm{mds}}\right)$ generally increased over the course of the flight (Fig. 3), averaging $4.4 \pm 9.3^{\circ}$ for wingbeat 1 and increasing to $78.3 \pm 13.7^{\circ}$ for wingbeat -1 . Both $\mathrm{TA}_{\mathrm{mds}}$ and

$22 \mathrm{BA}_{\mathrm{mds}}$ increased over $70^{\circ}$ from takeoff to landing. As a result, $T A_{m d s}$ relative to $B A_{m d s}$ did not vary significantly among wingbeats $(F=1.743, P=0.125)$. The tail was broadly spread throughout the flights, averaging $151 \pm 18^{\circ}$ over all wingbeats. Although mean tail spread at mid-downstroke increased 
somewhat from takeoff to landing, it did not vary significantly among wingbeats (repeated-measures, $F=1.745, P=0.125)$.

\section{Wing kinematics}

Similar patterns of downstroke sweep angle, wingbeat frequency, and thus, downstroke sweep velocity, were observed across the three phases of flight examined (Fig. 4). Downstroke sweep angle ( $\theta$ ) usually remained above $180^{\circ}$ throughout the flights (Fig. 4A). Values of $\theta$ above $180^{\circ}$ were reached for multiple reasons: the wingtip traveled medial to the wing root, the wingtips often crossed at USDS and DSUS, and because sweep angle as calculated here (in contrast to simple stroke amplitude) accounts for curvature in the wing stroke path. Values of $\theta$ differed significantly among wingbeats (repeated-measures, $F=8.669, P<0.001$ ) and were greatest during the first two wingbeats of takeoff

$12\left(203 \pm 5^{\circ}\right)$. During landing, $\theta$ increased from the midflight value of $176 \pm 17^{\circ}$ until reaching a maximum of $192 \pm 7^{\circ}$ at wingbeat -1 , after which it decreased significantly to $174 \pm 10^{\circ}$ during wingbeat 0 ( $t$-test, $P=0.002$ ). Wingbeat frequency (WBF) varied significantly among wingbeats (repeated-measures, $F=15.850, P<0.001)$. WBF declined steadily during takeoff, from $8.88 \pm 0.28 \mathrm{~s}^{-1}$ at wingbeat 1 to $7.68 \pm 0.56$

$16 \mathrm{~s}^{-1}$ at wingbeat 4 (Fig. 4B). During midflight, WBF averaged 6.57 $\pm 0.30 \mathrm{~s}^{-1}$, which was significantly lower than all other wingbeats except wingbeat 0 ( $t$-tests, $P=0.367 v s$. wingbeat $0, P<0.001$ vs. all other wingbeats). Similar to the pattern seen for $\theta$, WBF increased during landing, reaching a maximum at wingbeat $-1\left(7.78 \pm 0.25 \mathrm{~s}^{-1}\right)$ before decreasing significantly for wingbeat $0\left(6.81 \pm 0.67 \mathrm{~s}^{-1} ; P<0.001\right)$. Because $\theta$ and WBF followed similar patterns, downstroke sweep velocity $(\omega)$ also showed a similar pattern (Fig. 4C) and showed significant variation among wingbeats (repeated-measures, $F=25.064$,

$22 P<0.001)$. Values of $\omega$ declined during takeoff from $3.16 \pm 0.16^{\circ} \mathrm{ms}^{-1}$ for wingbeat 1 to $2.51 \pm 0.23^{\circ} \mathrm{ms}^{-1}$ for wingbeat 4. During landing, $\omega$ increased to a maximum at wingbeat $-1\left(2.45 \pm 0.11^{\circ} \mathrm{ms}^{-1}\right)$ before 24 decreasing significantly for wingbeat $0\left(2.11 \pm 0.19^{\circ} \mathrm{ms}^{-1} ; P=0.002\right)$. 
Figure 5 shows the path of the wingtip during an example takeoff, midflight, and landing of Bird

2 2. During most wingbeats of takeoff and landing, the birds' wingtips traced a figure-eight pattern relative to the shoulder in the lateral view. During midflight, the wingtips of most birds traced a path

4 around the shoulder, though some birds' wingtips still showed a figure-eight pattern. The compressed appearance of the wingtip path in the dorsal view during takeoff is due to the steep stroke plane angle

6 (SPA) relative to the horizontal plane. The wingtip traces from the lateral view illustrate the shift in the SPAs from negative during takeoff to positive during landing. Local stroke plane angle $\left(\mathrm{SPA}_{\text {loc }}\right)$ varied

8 significantly among wingbeats (repeated-measures, $F=219.309, P<0.001$ ) and was greater during landing wingbeats than during takeoff wingbeats $(P<0.001$ for all such comparisons; Fig. $6 \mathrm{~A})$. During takeoff, $\mathrm{SPA}_{\text {loc }}$ increased from $-60.8 \pm 5.3^{\circ}$ for wingbeat 1 to $-47.5 \pm 1.0^{\circ}$ for wingbeat 4 (Fig. $6 \mathrm{~B}$ ). During midflight, $\mathrm{SPA}_{\mathrm{loc}}$ averaged $-29.5 \pm 5.5^{\circ}$. During landing, $\mathrm{SPA}_{\mathrm{loc}}$ increased from $-0.9 \pm 1.7^{\circ}$ during wingbeat -3 to

$1217.3 \pm 7.4^{\circ}$ during wingbeat 0 . The local stroke plane angle relative to the body angle at mid-downstroke $\left(\mathrm{SPA}_{\text {relBA }}\right)$ was restricted to a much narrower range during flight, averaging $-48.2 \pm 7.0^{\circ}$ over all three modes of flight (mean of all flights; Fig. 6B). SPA ${ }_{\text {relBA }}$ differed significantly among wingbeats (repeated-measures, $F=5.947, P<0.001$ ), but post hoc comparisons did not show systematic differences related to flight mode. The global downstroke plane angle $\left(\mathrm{DSPA}_{\text {glob }}\right)$ showed changes similar to SPA $\mathrm{I}_{\text {loc }}$ increasing steadily from $-60.5 \pm 5.6^{\circ}$ at wingbeat 1 during takeoff to $13.1 \pm 7.0^{\circ}$ at wingbeat 0 during landing (Fig. 6B).

Wing protraction decreased slightly but not significantly during takeoff $(P>0.003$ for each comparison; Fig. $6 \mathrm{C}$ ) averaging $8.1 \pm 1.7 \mathrm{~cm}$ over all takeoffs, but increased during landing from $6.2 \pm 2.0$ $\mathrm{cm}$ during wingbeat -3 to $10.7 \pm 1.3 \mathrm{~cm}$ for wingbeat 0 . Wing protraction was significantly greater for 22 wingbeat 0 than for all other wingbeats ( $P \leq 0.002$ for each comparison), while during midflight it was significantly lower than for all other wingbeats $(2.7 \pm 2.5 \mathrm{~cm} ; P<0.001$ for each comparison). The vertical angles of the three wing planes (defined in Fig. 1) all increased more than $90^{\circ}$ from takeoff to landing 
(Fig. 6B). The plane angles did not differ significantly from each other in most instances ( $t$-tests; $P>0.008$

2 for 18 of 24 comparisons; $P \leq 0.002$ for arm wing vs. proximal and distal hand wings in wingbeat 4 , arm wing vs. proximal hand wing in wingbeats $-2,-1,0$, and proximal vs. distal hand wings in wingbeat -2 ). The angle of several features - stroke planes, wing planes, and tail - increased from takeoff to landing, in concert with the increase in body angle at mid-downstroke (Fig. 7). The local stroke plane

6 angle $\left(\mathrm{SPA}_{\mathrm{loc}}\right)$ was highly correlated with body angle $\left(R^{2}=0.950\right)$. Notably, the coefficient of the regression of SPA $\mathrm{Acc}_{\text {loc }}$ against body angle (0.99) reflects the uniformity of SPA $\mathrm{A}_{\text {relBA }}$ across wingbeats, and

8 the constant $(-48.0)$ reflects the typical value of $\mathrm{SPA}_{\text {relBA }}$ measured for individual wingbeats $\left(-48.2 \pm 7.0^{\circ}\right)$. Increases in wing plane angles were also highly correlated with increase in body angle (arm wing: $R^{2}=0.925$; proximal hand wing: $R^{2}=0.950$; distal hand wing: $R^{2}=0.957$ ). The changes in tail angle were also correlated with changes in body angle $\left(R^{2}=0.783\right)$.

\section{DISCUSSION}

In this study we sought to distinguish important differences in wing and body kinematics among takeoff, midflight, and landing in the pigeon, with the goal of interpreting these patterns in the context of their possible aerodynamic significance. During takeoff, we found that pigeons continued to accelerate after leaving the perch. Earls found that at the instant that starlings (Sturnus vulgaris) and quail (Coturnix coturnix) leave the ground during takeoff, they have already achieved $80-90 \%$ of the velocity they will reach by the following upstroke-downstroke transition (Earls, 2000). For hummingbirds taking off from a perch, this value is far less, 59\% (Tobalske et al., 2004). When the pigeons here broke contact with the perch, they had achieved $74 \pm 10 \%$ of the velocity reached by the

22 next upstroke-downstroke transition (USDS). Consequently, most of the acceleration into flight is the result of push-off from the legs. However, after the first takeoff wingbeat, the pigeons in this study 
legs to provide most of the acceleration for the first wingbeat, but the wings subsequently provided

2 significant acceleration to achieve the velocities of midflight. Bonser and Rayner found that when starlings land, they exert less force on the perch than when taking off, suggesting that starlings

4 decelerate prior to placing their feet on the perch (Bonser and Rayner, 1996). Consistent with this, we found that by the final USDS of landing, pigeons slowed to a quarter of the speed recorded prior to 6 landing, showing that most of the deceleration occurred during flight.

As expected, we found that downstroke amplitude and wingbeat frequency were greatest 8 during takeoff flight, consistent with earlier observations (Dial, 1992). Sweeping the wing through a greater amplitude increases the amount of air that the wing accelerates, increasing its lift and drag. But contrary to our expectations, downstroke sweep velocity was highest during takeoff, rather than landing. This suggests that the extra drag on faster-moving wings is insufficient to affect these

12 maneuvers for the pigeon. If the drag were greater, we would expect that the birds would move the wings faster during landing or slower during takeoff. Consequently, changes in downstroke wing sweep

14 velocity likely reflect the need to adjust lift. During takeoff, the need to produce more lift may compensate for the cost of extra drag, leading to a greater sweep velocity. Our observations also suggest that increased lift requirements during takeoff may play a greater role in determining wing sweep angle and velocity than the need for increased drag to slow the bird during landing. As past workers have noted (e.g., Tobalske and Dial, 1996; Usherwood et al., 2005), the tail is broadly spread in pigeons during flight at low speeds, including the takeoff and landing phases. But contrary to our expectations, the tail was not spread significantly more during landing to increase drag, nor significantly less during takeoff to decrease drag. This suggests that pigeons do not use tail spread

22 as a mechanism to modify the magnitude or orientation of aerodynamic force during takeoff and landing. 
We found that the wing was most protracted during the end of landing, when flight velocity was

2 lowest and body angle highest. A more protracted wing posture is also observed during the slow descending flights of pigeons (Berg and Biewener, 2008), suggesting that pigeons maintain more

4 protracted wing positions during slower flights with steep body angles. By shifting the plane of lift generation produced by the wings farther above the body's center of mass through a more protracted wing stroke, pigeons may increase their stability during such flight behaviors.

Surprisingly, several kinematic parameters rotated through large angles in concert from takeoff

8 to landing (Fig. 7). We found that body angle, tail angle, and wing plane angles all increased more than $70^{\circ}$ from takeoff to landing (Fig. 3). These coordinated changes in body and wing orientation likely reduced the drag on the bird during takeoff to assist in acceleration, and increased drag during deceleration and landing. The figure-eight shape of the wingtip paths in the lateral view during both

12 takeoff and landing was similar to that observed in pigeons during slow ascending, level, and descending flight (Tobalske and Dial, 1996; Berg and Biewener, 2008), but the rotation of the figure-eight pattern makes apparent the shift in stroke plane angle (SPA) from a steep downward tilt during takeoff to an upward tilt during landing (Fig. 5). Similar to the rotation observed in body, wing, and tail angles, both the local stroke plane angle $\left(\mathrm{SPA}_{l o c}\right)$ and the global downstroke plane angle $\left(\mathrm{DSPA}_{\text {glob }}\right)$ increased over $70^{\circ}$ (Fig. 6B). The downward tilt of the SPA $\mathrm{Alc}_{\text {oc }}$ during takeoff would have increased the forward thrust by pushing more air rearward and thus directing the aerodynamic force more forward. The upward tilt of the $\mathrm{SPA}_{\mathrm{loc}}$ during landing would have pushed more air forward, directing the aerodynamic force more rearward. Although the shift in stroke plane angle was expected, we did not anticipate the highly correlated increases in body, tail, and wing plane angles. It thus appears that pigeons rotate several

22 features - body, wing, tail, and stroke plane - to decrease drag and increase thrust during takeoff, and to increase drag and decrease forward thrust during landing; yet, all of these changes are effected by 24 simply rotating the body as a whole (Fig. 7). Importantly, this finding also suggests that changes in 
muscle activation and contractile patterns needed to control wing motion relative to the body are likely

2 to be small across these changes in flight mode, potentially simplifying the motor control requirements for undergoing takeoff and landing flight.

The relatively small degree of change in kinematic parameters with respect to the body axis implies that only slight changes are necessary to pitch the body of the bird up or down. Based on the results presented here, we estimated the maximum moment arm necessary to pitch the body of a pigeon upward during the transitions from takeoff to midflight and from midflight to landing. We assumed angular acceleration of the body during steady flight to be zero and used the body angle and downstroke duration measurements at wingbeat 4 (near the end of takeoff), midflight, and wingbeat -3 (near the beginning of landing) to estimate maximum angular accelerations. Moment of inertia for pitch of a pigeon was estimated to be $0.00144 \mathrm{~kg} \mathrm{~m}^{2}$ by adjusting the measurement of Ros and Biewener

12 (unpublished results) for the mass of the birds in this study. Mean body weight, $4.2 \mathrm{~N}$, was used as a minimum value for aerodynamic force. We found that the maximum moment arm from the aerodynamic force vector to the center of mass necessary to pitch the body upward was $1.4 \mathrm{~mm}$ from takeoff to midflight and $1.7 \mathrm{~mm}$ from midflight to landing. These remarkably small moment arms suggest that only slight adjustments in kinematics and muscle function are necessary to pitch the body during the transitions between flight phases. Consequently, because the stroke plane, wing plane, and tail angles all rotate in concert with the body angle, very subtle changes in kinematics are sufficient to produce major shifts in flight mode.

To summarize, we found that the pigeon's stroke plane is tilted steeply downward to accelerate the bird during takeoff, but shifts to tilt upward to slow the bird during landing. Importantly, most of 22 the change in local and global stroke plane angles is achieved by body rotation, while the stroke plane angle relative to the body remains uniform across the different phases of flight. Wing plane and tail angles also rotate from takeoff to landing, in concert with the body angle. Estimates of the moment arm 
required to rotate the body suggest that very subtle changes in kinematics can pitch the bird upward

2 during flight. The positioning of the wings, tail, and body all appear to contribute to reducing drag or increasing thrust during takeoff, and to increasing drag during landing. The high correlations between

4 body angle and stroke plane, wing plane, and tail angles suggest that instead of modifying body posture and stroke orientation, pigeons simply rotate the entire body and thereby direct aerodynamic force more forward during takeoff and more rearward during landing. Analysis of fluid dynamics around pigeons during these maneuvers is needed to confirm these hypotheses and is currently underway.

8 Study of muscle function during flight is also currently underway to investigate whether the simplicity of this mechanism simplifies the muscle control necessary during takeoff and landing.

We would like to thank the many people at the Concord Field Station who were involved with these

12 experiments, particularly Pedro Ramirez for caring for the pigeons. This work was supported in part by NSF grant: IOS-0744056.

\section{List of symbols}

whole-body acceleration in the $x$-direction over the course of one wingbeat body angle at mid-downstroke downstroke plane angle in the global coordinate system downstroke-upstroke transition

$24 \mathrm{SPA}_{\text {relBA }}$ $T A_{\text {mds }}$ kinetic energy potential energy stroke plane angle local stroke plane angle

$26 \quad \begin{aligned} & \text { USDS } \\ & \mathrm{V}_{x}\end{aligned}$
difference between body angle and SPA $A_{\text {loc }}$ tail angle during mid-downstroke upstroke-downstroke transition whole-body velocity in the $x$-direction

$\begin{array}{lll}\omega & \omega & \text { wingbeat frequency } \\ 30 & \theta & \text { downstroke sweep velocity } \\ & & \text { downstroke sweep angle }\end{array}$




\section{REFERENCES}

Askew, G. N., Marsh, R. L. and Ellington, C. P. (2001). The mechanical power output of the flight muscles of blue-breasted quail (Coturnix chinensis) during take-off. J Exp Biol 204, 3601-3619.

Berg, A. M. and Biewener, A. A. (2008). Kinematics and power requirements of ascending and descending flight in the pigeon (Columba livia). J Exp Biol 211, 1120-1130.

Biewener, A. A., Corning, W. R. and Tobalske, B. W. (1998). In vivo pectoralis muscle force-length behavior during level flight in pigeons (Columba livia). J Exp Biol 201, 3293-3307.

Bonser, R. H. C. and Rayner, J. M. V. (1996). Measuring leg thrust forces in the common starling. J Exp Biol 199, 435-439.

Brown, R. H. J. (1948). The flight of birds: the flapping cycle of the pigeon. J Exp Bio/ 25, 322-333.

Dial, K. P. (1992). Activity patterns of the wing muscles of the pigeon (Columba livia) during different modes of flight. J Exp Zool 262, 357-373.

Earls, K. D. (2000). Kinematics and mechanics of ground take-off in the starling Sturnis vulgaris and the quail Coturnix coturnix. J Exp Biol 203, 725-739.

Green, P. R. and Cheng, P. (1998). Variation in kinematics and dynamics of the landing flights of pigeons on a novel perch. J Exp Biol 201, 3309-3316.

Hatze, H. (1988). High-precision three-dimensional photgrammetric calibration and object space reconstruction using a modified DLT approach. J Biomech 21, 533-538.

Hedrick, T. L. (2008). Software techniques for two- and three-dimensional kinematic measurements of biological and biomimetic systems. Bioinspiration and Biomimetics 3, 1-6.

Hedrick, T. L., Usherwood, J. R. and Biewener, A. A. (2004). Wing inertia and whole-body acceleration: an analysis of instantaneous aerodynamic force production in cockatiels (Nymphicus hollandicus) flying across a range of speeds. J Exp Biol 207, 1689-1702.

Rice, W. R. (1989). Analyzing tables of statistical tests. Evolution 43, 223-225.

Rosen, M., Spedding, G. R. and Hedenstrom, A. (2004). The relationship between wingbeat kinematics and vortex wake of a thrush nightengale. J Exp Biol 207, 4255-4268.

Simpson, S. F. (1983). The Flight Mechanism of the Pigeon Columbia-Livia During Take-Off. Journal of Zoology 200, 435-443.

Tobalske, B. W. and Dial, K. P. (1996). Flight kinematics of black-billed magpies and pigeons over a wide range of speeds. J Exp Biol 199, 263-280.

Tobalske, B. W. and Dial, K. P. (2000). Effects of body size on take-off flight performance in the Phasianidae (Aves). J Exp Biol 203, 3319.

Tobalske, B. W. and Biewener, A. A. (2008). Contractile properties of the pigeon supracoracoideus during different modes of flight. J Exp Biol 211, 170-179.

Tobalske, B. W., Hedrick, T. L. and Biewener, A. A. (2003). Wing kinematics of avian flight across speeds. Journal of Avian Biology 34, 177-184.

Tobalske, B. W., Altshuler, D. L. and Powers, D. R. (2004). Take-off mechanics in hummingbirds (Trochilidae). J Exp Biol 207, 1345-1352.

Usherwood, J. R., Hedrick, T. L., McGowan, C. P. and Biewener, A. A. (2005). Dynamic pressure maps for wings and tails of pigeons in slow, flapping flight, and their energetic implications. J Exp Biol 208, 355-369. 
Fig. 1. Calculation of the wing and tail angles (A) and the local stroke plane angle (SPA loc; B). Triangles

2 defining the wing and tail planes were based on the digitized points $(\mathrm{A})$. The vertical wing and tail angles were calculated as the angle between the plane and the horizontal. The tail spread was calculated using

4 the assumption that the tail formed a wedge of a circle, as described in the text. For each wingbeat, the linear regression of the $x$ - and $z$-coordinates of the wingtip position relative to the shoulder was calculated (B). SPA $A_{\text {loc }}$ is the angle corresponding to the slope of the linear regression. SPA $A_{\text {loc }}$ is negative when the wingtip moves forward and downward during downstroke (as illustrated here), and positive

8 when the wing moves forward and upward during downstroke (as in landing). The global downstroke plane angle (DSPA $\left.{ }_{\text {glob }}\right)$ was calculated in a similar manner, using wingtip position in the global coordinate system instead.

12 Fig. 2. Whole-body kinematics: individual means of bird velocity in the $x$-direction $\left(V_{x}\right)$ at the upstroke-downstroke transitions (USDS; A); grand means of $\mathrm{V}_{\mathrm{x}}$ at USDSs and acceleration in the

$14 x$-direction over the course of the wingbeat $\left(\mathrm{A}_{x, w b} ; B\right)$; and kinetic and potential energies (KE and PE; $C$ ). Points for $\mathrm{V}_{x}, K E$, and PE indicate data for each USDS. Points for $A_{x, w b}$ indicate data for each wingbeat. Colors represent individual birds. $V_{x}$ increased for all individuals after leaving the perch during takeoff (A). During landing, $\mathrm{V}_{x}$ decreased for all individuals prior to encountering the perch but was more variable than during takeoff. $A_{x, w b}$ was greatest during the second wingbeat of takeoff, and became increasingly negative during landing. As expected, KE followed the pattern observed for $V_{x}$, and PE did not change during takeoff or landing (C). Thus, in the flights analyzed, there was no tradeoff between KE and PE.

Fig. 3. Body and tail angles at mid-downstroke for each wingbeat. Body angle and vertical tail angle 24 both increase continually throughout takeoff and landing, with total increases of $85^{\circ}$ and $74^{\circ}$, 
respectively. Consequently, tail angle relative to body angle does not differ significantly among

2 wingbeats ( $P \geq 0.004$ for all comparisons, non-significant by sequential Bonferroni). Tail spread increased somewhat from takeoff to landing, but did not vary significantly among wingbeats (repeated-measures,

$4 \quad F=1.745, P=0.125)$.

6 Fig. 4. Downstroke sweep angle (A), wingbeat frequency (WBF; B), and downstroke sweep velocity (C) for each wingbeat. Downstroke sweep angle, a measure of wingbeat amplitude, is greatest during

8 takeoff (A). Downstroke sweep angle remains consistent during takeoff, but increases during landing before dropping at the final wingbeat. WBF declines during takeoff, but also increases during landing before dropping at the final wingbeat (B). Downstroke sweep velocity thus follows a similar pattern to both downstroke sweep angle and WBF (C).

12

Fig. 5. Example kinematic traces for takeoff, midflight, and landing for Bird 2. Arrows indicate the

14 direction of wingtip movement during downstroke. Top panels show wingtip position relative to the shoulder for each wingbeat. Grey crosses indicate the location of the shoulder when the origin is not shown. Lower panels show wingtip position in the global coordinate system, with tick marks indicating distance from the perch. The traces illustrate the changes in stroke planes from negative angles during 18 takeoff and midflight to positive angles during landing. The steeply-tilted stroke planes during takeoff account for the compressed appearance of the wingtip path in the dorsal view during takeoff. These trends in stroke plane angle are evident for both local and global coordinate systems.

22 Fig. 6. Wing kinematics: individual means of local stroke plane angle (SPA $\left.\mathrm{S}_{\text {loc; }} \mathrm{A}\right)$, grand means of stroke plane angles and wing plane angles at mid-downstroke (B), and wing protraction (C). (A) For all

24 individuals, SPA $\mathrm{l}_{\mathrm{loc}}$ increased during takeoff and landing, changing sign from negative during takeoff to 
positive by the end of landing. Colors represent individual birds, with open circles at midflight

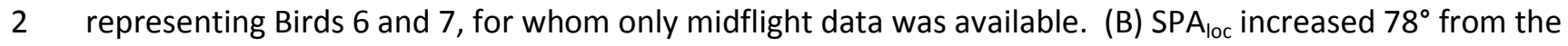
first wingbeat to the last, corresponding with the $85^{\circ}$ increase in body angle (Fig. 3). The $S P A_{\text {loc }}$ relative

4 to the body angle $\left(\mathrm{SPA}_{\text {relBA }}\right)$ thus changes relatively little during these flights. Downstroke plane angle in the global reference frame $\left(\mathrm{DSPA}_{\mathrm{glob}}\right)$ closely followed $\mathrm{SPA}_{\text {loc }}$. The vertical angles of the wing planes at

6 mid-downstroke all increased during takeoff and landing and were generally not significantly different from each other (see text for explanation of statistics). (C) Protraction of the wing, calculated as the

8 distance between the local stroke plane and the shoulder, did not change significantly during takeoff ( $P>0.003$ for each comparison), but increased during landing and by wingbeat 0 was greater than during all other wingbeats ( $P \leq 0.002$ for each comparison).

12 Fig. 7. Scatter plots and least-square linear regressions of stroke plane, wing plane, and tail angles plotted against body angle at mid-downstroke. Labeled brackets indicate body angle ranges for each

14 mode of flight. All of these angles increased similarly from takeoff to landing. Increases in local stroke plane angle (SPA $\left.{ }_{l o c}\right)$ and vertical wing plane angles were highly correlated with increases in body angle $16\left(R^{2}>0.92\right.$ for each). In addition, the coefficient of 0.99 in the regression equation for SPA $\mathrm{I}_{\text {loc }}$ reflects the uniformity of the $\mathrm{SPA}_{\text {loc }}$ relative to body angle. Tail angle at mid-downstroke $\left(\mathrm{TA}_{\mathrm{mds}}\right)$ was also correlated with body angle $\left(R^{2}=0.783\right)$. By controlling the orientation of the body as a whole, pigeons control the stroke plane, wing plane, and tail angles, thereby adjusting thrust and drag production and the direction of aerodynamic force. 

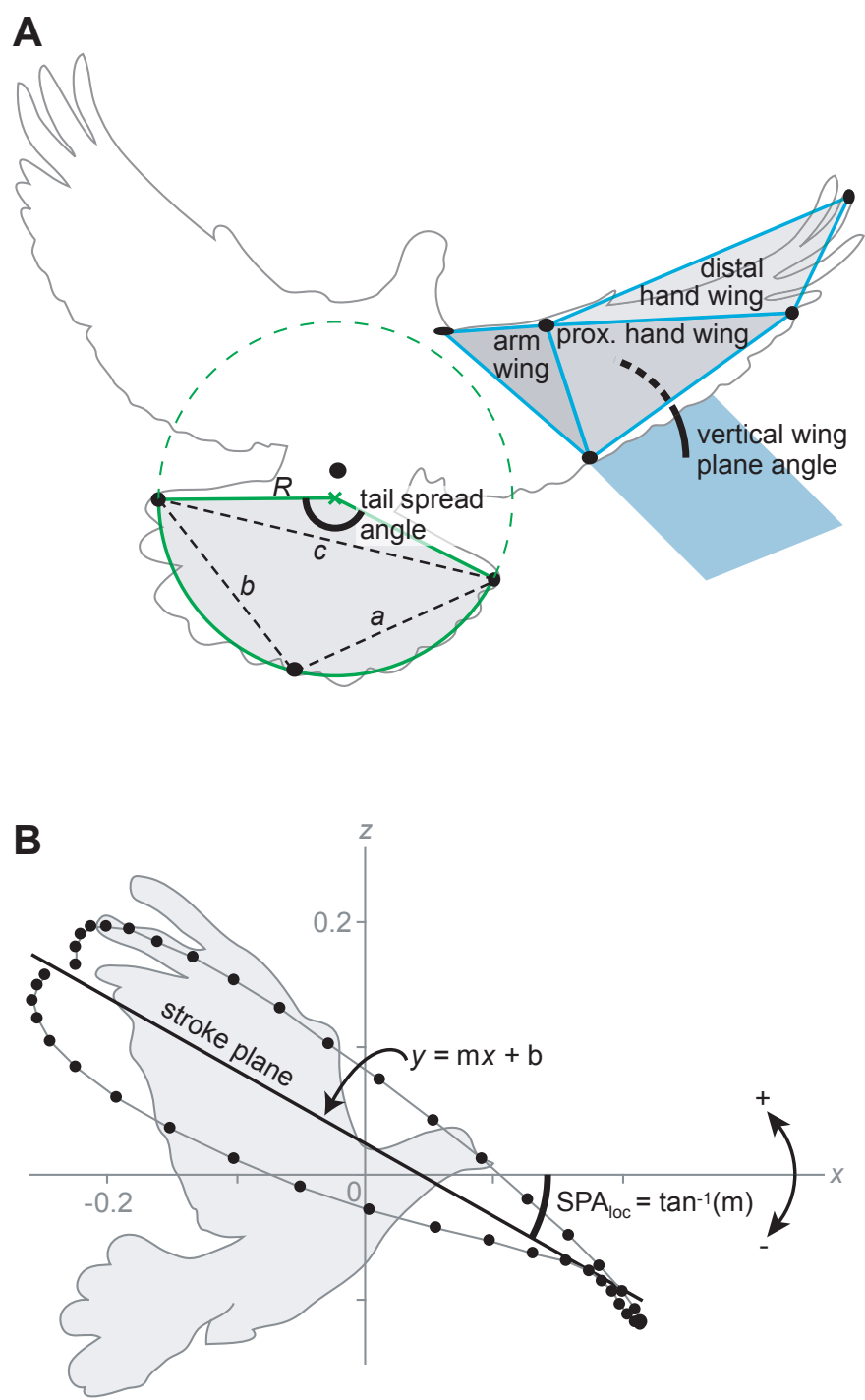

Figure 1 

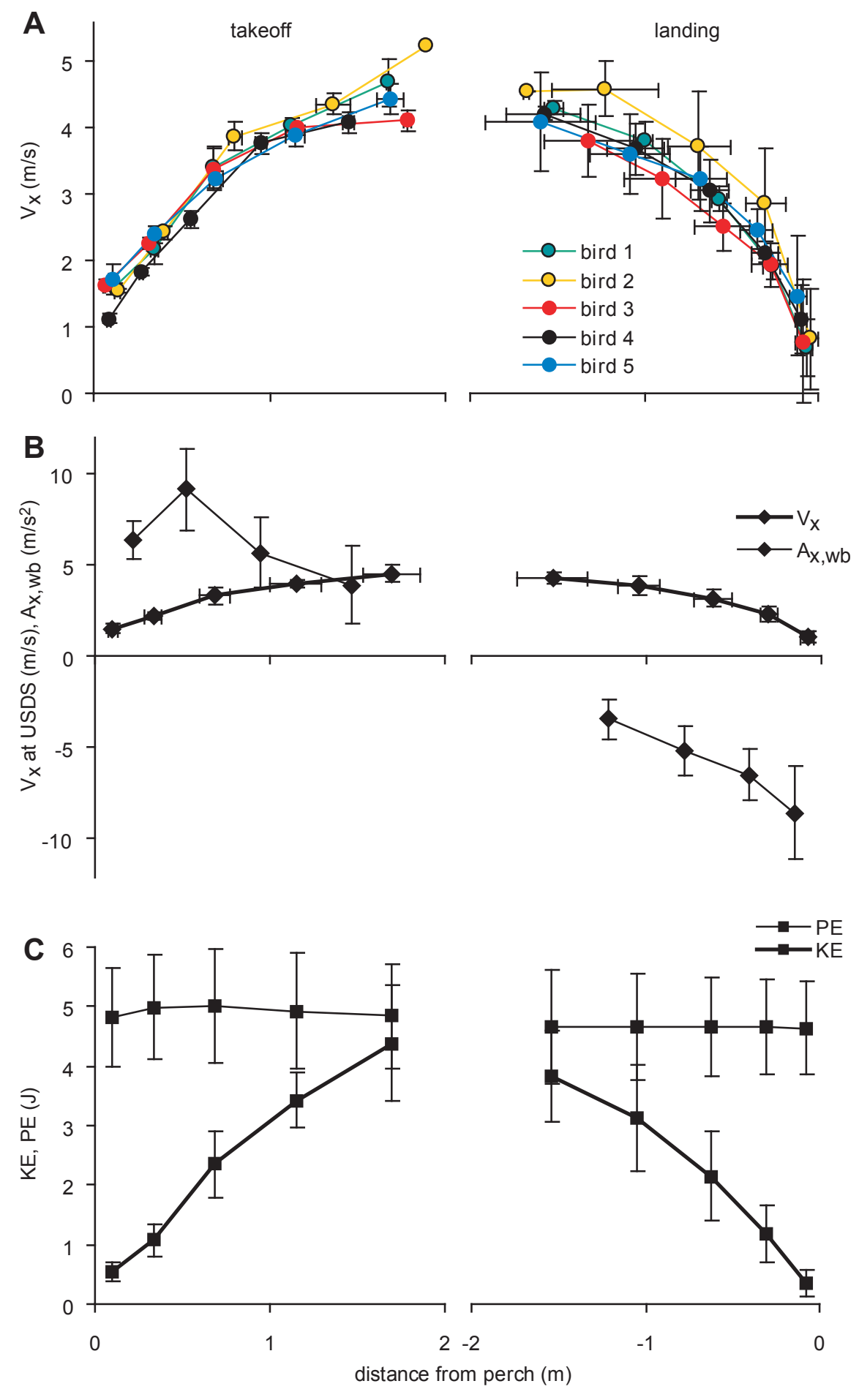

Figure 2 


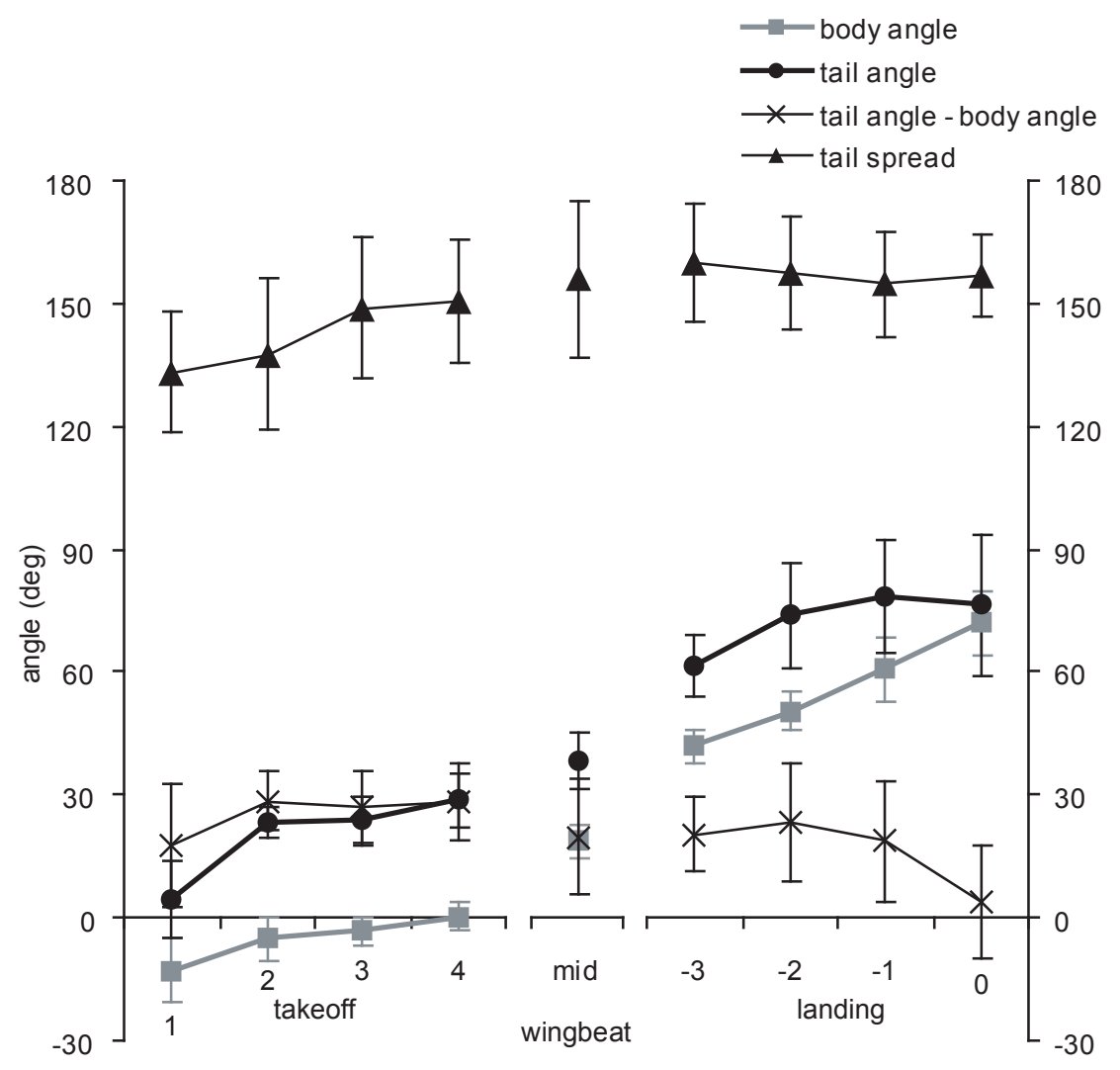

Figure 3 

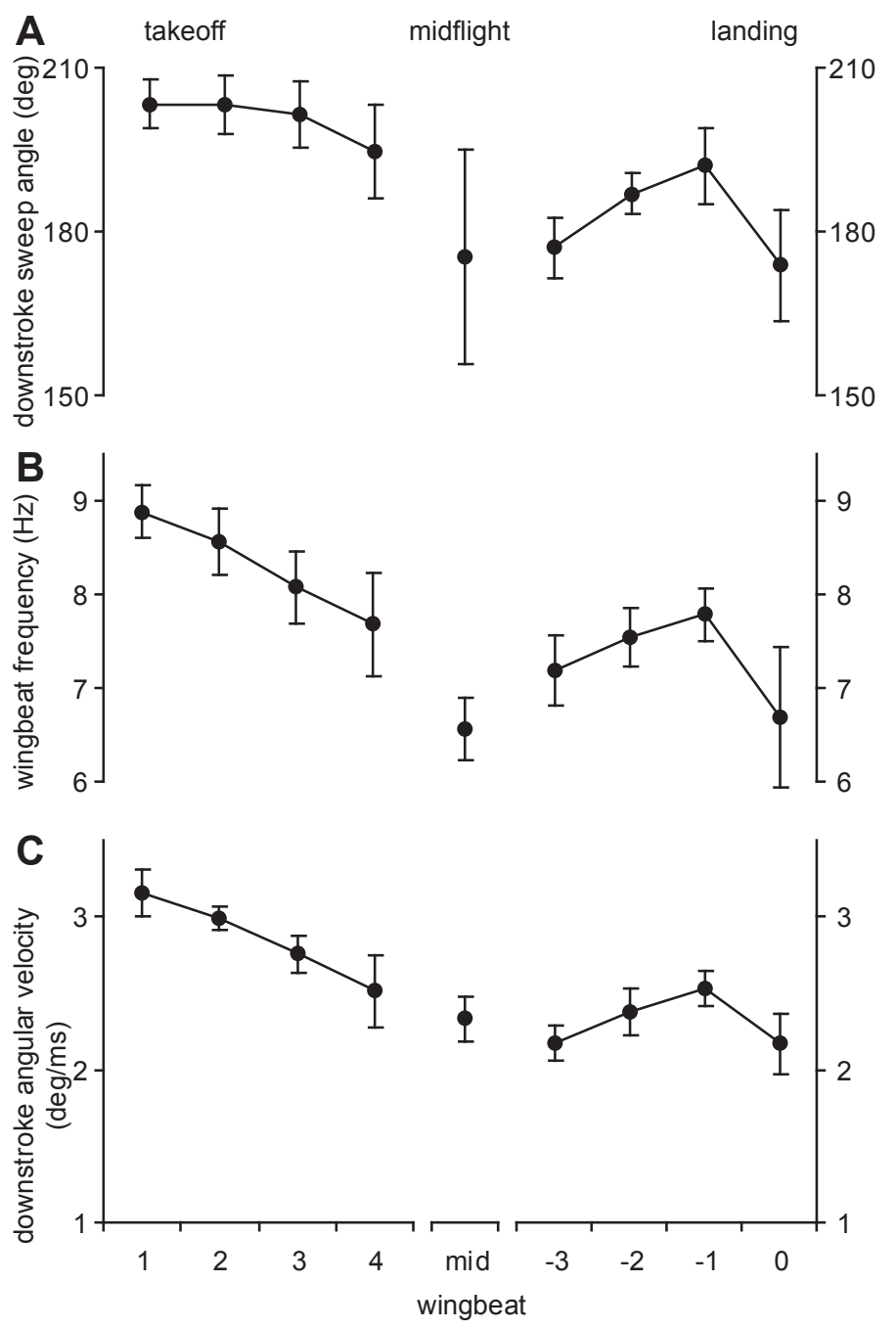

Figure 4 

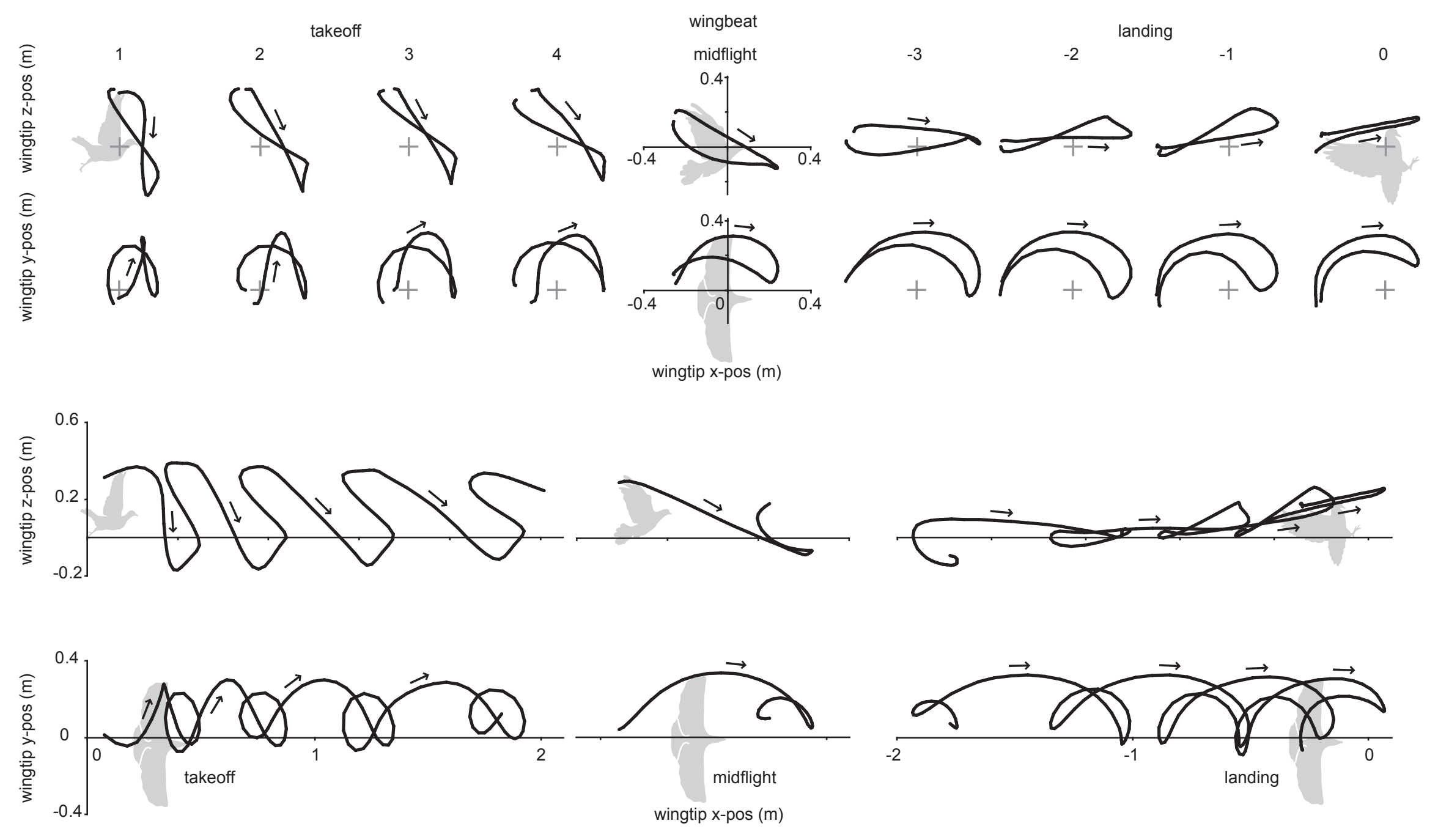

Figure 5 

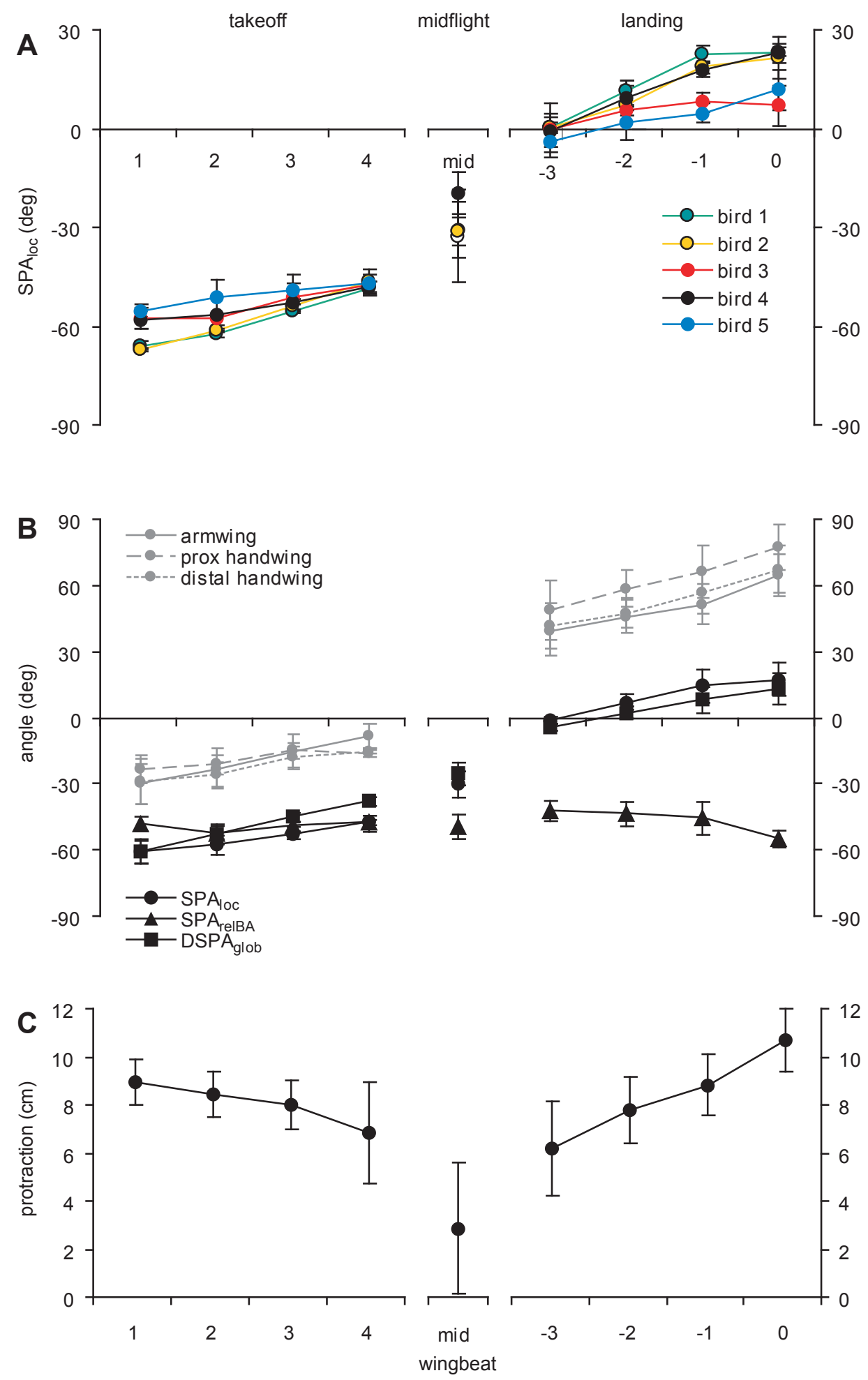

Figure 6 


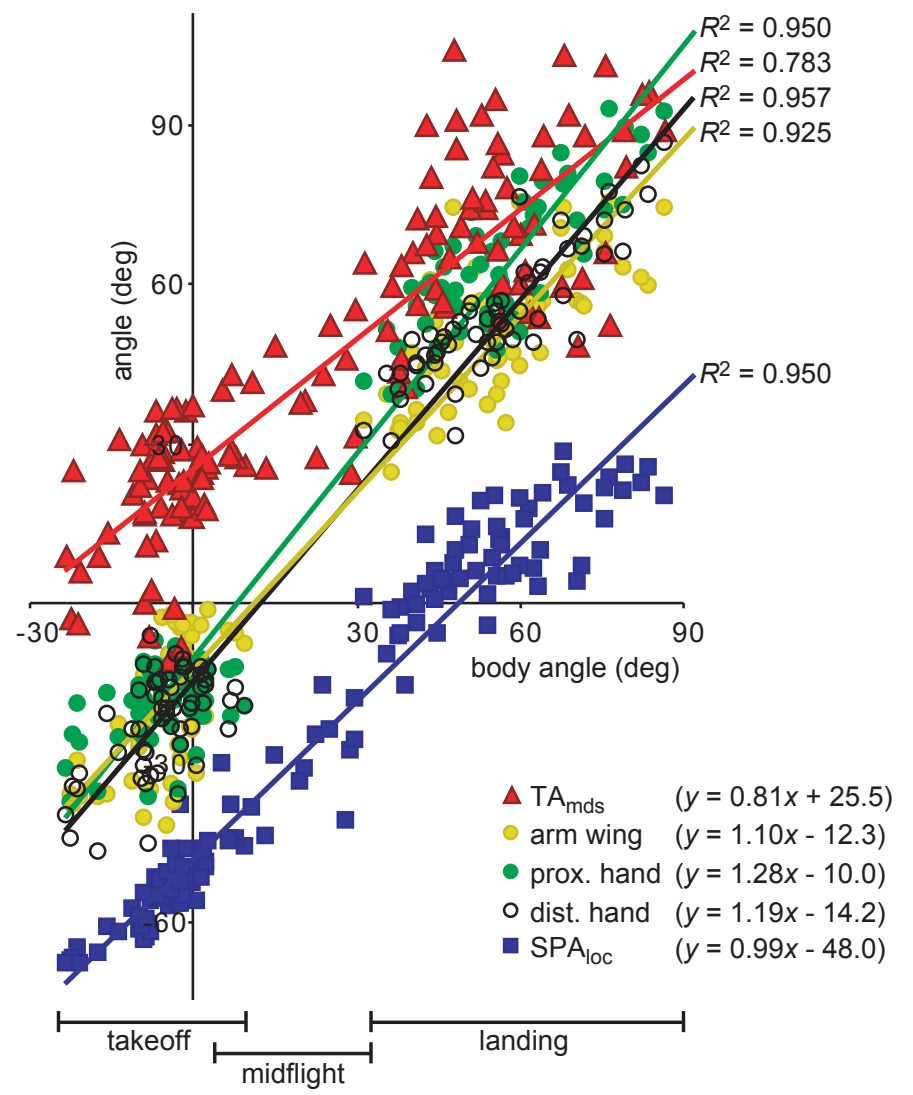

Figure 7 